\title{
STATIONARY BINNIE WAVES NEAR RESONANCE
}

\author{
BY \\ SONGPING ZHU \\ University of Wollongong, Wollongong, N.S.W. 2500, Australia
}

\begin{abstract}
Stationary waves in an open channel with corrugated side walls possess resonant speeds at or near which the prediction of free surface waves by the linear approximation fails. However, if the nonlinear terms in the Euler equation and boundary conditions are kept, a finite amplitude of these stationary waves can be determined. In this paper, such a nonlinear approach is presented, and the amplitude of the waves near the resonant speeds is determined to be of the order of the one-third power of the side-wall corrugation amplitude.
\end{abstract}

Introduction. The problem of resonance considered in this paper was first mentioned, but not solved, by Yih when dealing with stationary water waves in a channel induced by vertical corrugated side-walls [11] and stationary waves in a meandering stream [12]. These water waves, which have longitudinal wavelengths equal to the wavelength of the wall corrugation, and transverse wave-numbers as well, were first observed by Binnie [3] in a conduit with corrugated walls. Yih [11] gave an analytical solution to the stationary waves, which he called Binnie waves, although the waves discovered by Binnie also included waves moving upstream into the contraction which led from the supply reservoir. Yih [11] took the waviness of the side walls fully into account in his solution and revealed the mechanism by which the stationary waves were created. The stationary waves consist of infinitely many wave components, each with a different transverse wave-number. However, these linear solutions fail as the upstream velocity takes critical values, namely, when its component normal to the slanted waves is equal to the wave speeds in quiet water [11, 12]. The failure of the linear solution is inevitable as resonance occurs when these critical upstream velocities are reached.

The study of near-resonance problems of a water system involving a free surface and some sort of external oscillating force is of considerable practical importance. With the frequency of the oscillating force being near the so-called "cut-off" frequency, the excitation usually produces large responses (e.g., see Aranha et al. [1]). Such large responses can be found in a number of cases discussed previously in the literature. For example, Lamb [9] treated surface waves caused by a flow over a 
wavy bottom and encountered resonance as infinite amplitude was found at a critical Froude number. When discussing the failure of the solution near the resonance, Lamb conjectured that "To obtain an intelligible result in this case, we should be compelled to take special account of dissipative forces." However, later works showed that this is one of the very few places in Lamb's book to be in error. The resonant oscillations produced in a gas-filled tube closed at one end by a rigid barrier, and at the other by a vibrating piston were investigated by Betchov [2], Chu and Ying [4], and Chester [5]. They concluded that the oscillations near resonance appeared to be governed by nonlinear effects. Chester [5] presented a theoretical solution, by taking the nonlinear effects into account, of the problem of the unsteady surface oscillations in a finite tank under prescribed horizontal sinusoidal displacement. His result was compared with the experimental results, and reasonable agreement between the theory and experiment was found. Ockendon and Ockendon [10] extended Chester's analysis and found an explicit asymptotic solution. An asymptotic expansion with multiple scales was adopted by Jones [8] to deal with the resonance in a long deep channel with cross waves. Dagan and Miloh [6] showed that the resonance, occurring when a flow past a body that moves at a constant speed and simultaneously performs an oscillation near the free surface, can be removed by considering higherorder free-surface nonlinear effects. Ellermeier [7] challenged Lamb's [9] conjecture after carefully taking nonlinearity into consideration and successfully determining the amplitude of the free surface waves induced by a flow over a wavy bottom. All these studies have demonstrated the importance of including the nonlinear effects rather than the "dissipative forces" for the free-surface oscillation near resonance.

The aim of this paper is to present a solution to the problem of a layer of water flowing through a channel with corrugated side walls near critical speeds. In the following sections, Yih's solution [11] will first be shown to be a natural consequence of the first-order solution as an asymptotic expansion is applied to the nonlinear governing differential system in the water channel. To suppress the resonance near the critical flow speed, the expansion is carried out further, up to the third order of a small quantity $\varepsilon$, which is directly related to the amplitude of the corrugated side walls. It will be shown that the amplitude of the free surface waves, near the primary critical mean-flow speed (those found in the first-order approximation), is of the order of the side-wall corrugation amplitude to the one-third power. Higher-order resonances may exist as well, and their analysis becomes increasingly complicated. However, the chance of these higher-order resonances being excited becomes less and less as the order increases. They are therefore not as important as the primary resonances treated herein.

1. Formulation of the problem. A layer of incompressible, inviscid fluid with mean layer thickness $h$ flows through an open channel with corrugated side walls described by $y^{*}= \pm L+A \cos k^{*} x^{*}$, where $k^{*}$ is the dimensional wave number of the channelwidth variation, $L$ is the mean half-width of the channel and $A(A \ll L)$ is the amplitude of the corrugated side walls. The coordinate system is chosen such that the $(x, y)$-plane is the mean position of the free surface when the fluid is tranquil and the $z$-axis points vertically upward as shown in Fig. 1. 


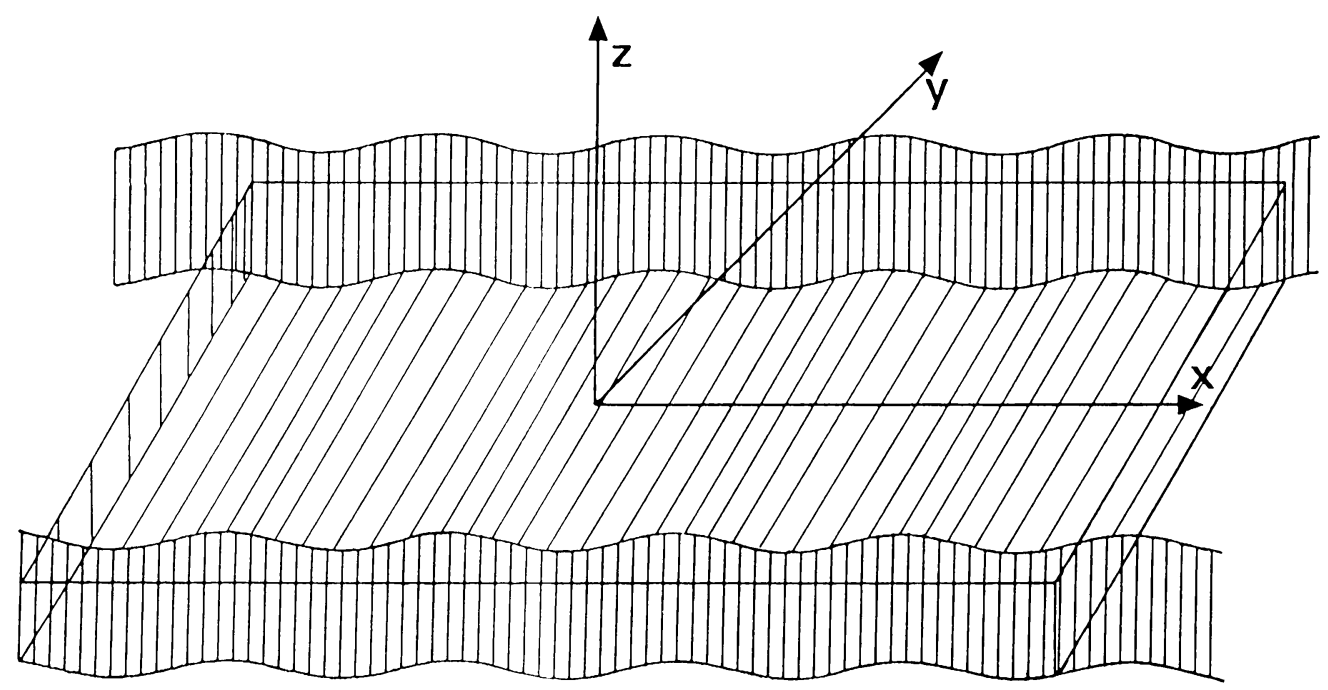

FIG. 1. A schematic view of the water channel and coordinate system.

The theory is constructed on the assumption of irrotational flow. If the upstream velocity $U$ is adopted as the velocity scale and $L$ as the length scale, the dimensionless governing differential equation for the potential flow is the Laplace equation:

$$
\Delta \phi=\phi_{x x}+\phi_{y y}+\phi_{z z}=0,
$$

where $\phi$ is the potential function from which the velocity components are found as follows:

$$
(u, v, w)=\left(\phi_{x}, \phi_{y}, \phi_{z}\right) .
$$

In (1) and (2), subscripts denote partial differentiation, and $x, y, z$ are dimensionless coordinates measured, respectively, along the channel center line, across the channel and in the direction opposite to the gravitational acceleration $g$.

Let the dimensionless displacement of the free surface above its mean position be denoted by $\zeta$. Then, with the flow steady, the kinematic condition for the free surface is

$$
u \zeta_{x}+v \zeta_{y}=w
$$

and the Bernoulli equation written for the free surface is

$$
u^{2}+v^{2}+w^{2}+2 F^{-2} \zeta=\text { const. }
$$

where $F$ is the Froude number defined as $U / \sqrt{g L}$.

Upon eliminating $\zeta$ from (3) and (4), the boundary condition on the free surface becomes

$$
\left(\phi_{x} \frac{\partial}{\partial x}+\phi_{y} \frac{\partial}{\partial y}\right)\left(\phi_{x}^{2}+\phi_{y}^{2}+\phi_{z}^{2}\right)+2 F^{-2} \phi_{z}=0 .
$$

If the dimensionless mean depth is denoted by $d(=h / L)$, the boundary condition expressing zero fluid flux at the bottom of the channel is

$$
\phi_{z}=0 \quad \text { at } z=-d \text {. }
$$


The boundary conditions on the side walls are

$$
\phi_{n}=0 \quad \text { at } y= \pm 1+\frac{A}{L} \cos k x,
$$

where $k$ is the dimensionless wave number of the side-wall corrugation and $n$ is the direction normal to the side wall. Equations (1), (5), (6), and (7) govern the fluid motion in the channel, even though the flow along the channel is yet to be built into the system.

2. Solution. Following the conformal mapping adopted in Yih [11], the governing differential equation as well as the boundary conditions can be transformed into a space where the $\gamma$-plane $\left(\gamma=\alpha+i \beta, i^{2}=-1\right)$ replaces the $w$-plane $(w=x+i y)$ according to the transformation function

$$
w=\gamma+a \sin \gamma
$$

where $a=A /(L \sinh k)$, the dimensionless amplitude of the wavy side walls. After the transformation, the governing differential equation in the new coordinate system (shown in Fig. 2 with $z$-coordinate unchanged) becomes

$$
\frac{1}{J}\left(\phi_{\alpha \alpha}+\phi_{\beta \beta}\right)+\phi_{z z}=0
$$

which is to be subject to the boundary conditions

$$
\begin{gathered}
\frac{1}{J}\left(\phi_{\alpha} \frac{\partial}{\partial \alpha}+\phi_{\beta} \frac{\partial}{\partial \beta}\right)\left[\frac{1}{J}\left(\phi_{\alpha}^{2}+\phi_{\beta}^{2}\right)+\phi_{z}^{2}\right]+2 F^{-2} \phi_{z}=0 \text { at } z=\zeta, \\
\phi_{\beta}=0 \quad \text { at } \beta= \pm 1, \\
\phi_{z}=0 \quad \text { at } z=-d,
\end{gathered}
$$

where $J$ is the Jacobian of the transformation given in [11] as

$$
J=\frac{\partial(x, y)}{\partial(\alpha, \beta)}=1+2 a k \cos k \alpha \cosh k \beta+a^{2} k^{2}\left(\cos ^{2} k \alpha+\sinh ^{2} k \beta\right) .
$$

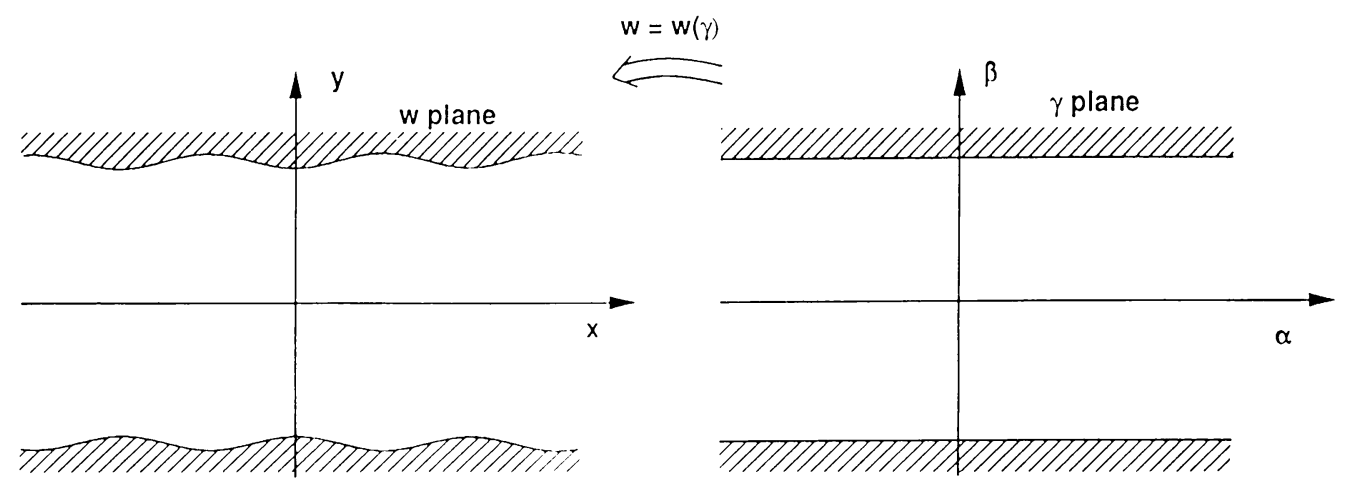

FIG. 2. The mapping between $w$-plane and $\gamma$-plane. 
After expanding $\phi, a$, and $\zeta$ in terms of a small quantity $\varepsilon$, which is a small number directly related to the amplitude of the side-wall corrugation, ${ }^{1}$ one obtains

$$
\begin{aligned}
& a=\varepsilon a_{1}+\varepsilon^{2} a_{2}+\varepsilon^{3} a_{3}+\cdots, \\
& \phi=\phi_{0}+\varepsilon \phi_{1}+\varepsilon^{2} \phi_{2}+\varepsilon^{3} \phi_{3}+\cdots, \\
& \zeta=\varepsilon \zeta_{1}+\varepsilon^{2} \zeta_{2}+\varepsilon^{3} \zeta_{3}+\cdots .
\end{aligned}
$$

Equations (14), (15), and (16) can be inserted into (8)-(12) to form the differential equation and boundary conditions order by order after the boundary conditions on the free surface are expanded in a Taylor series. The $\phi_{0}$ term in the above expansion is nothing but the potential function corresponding to the uniform flow, i.e., $\phi_{0}=\alpha$. The first-order differential equation and boundary conditions are

$$
\begin{gathered}
\phi_{1 \alpha \alpha}+\phi_{1 \beta \beta}+\phi_{1 z z}=0 \\
\phi_{1 \alpha \alpha}+2 F^{-2} \phi_{1 z}=-a_{1} k^{2} \sin k \alpha \cosh k \beta \quad \text { at } z=0 \\
\phi_{1 \beta}=0 \quad \text { at } \beta= \pm 1 \\
\phi_{1 z}=0 \quad \text { at } z=-d,
\end{gathered}
$$

which are identical to the governing differential equations in [11].

The second-order differential equation and boundary conditions are nonhomogeneous because the product of the terms of the first-order solution becomes the nonhomogeneous terms on the right-hand side of the second-order equations. They are, by collecting the terms of order $\varepsilon^{2}$,

$$
\begin{gathered}
\phi_{2 \alpha \alpha}+\phi_{2 \beta \beta}+\phi_{2 z z}=-2 k a_{1} \cos k \alpha \cosh k \beta \phi_{1 z z} \\
\phi_{2 \alpha \alpha}+F^{-2} \phi_{2 z}=-[ \\
\frac{\partial}{\partial \alpha}\left(\phi_{1 \alpha}^{2}+\phi_{1 \beta}^{2}+\frac{1}{2} \phi_{1 z}^{2}\right)+\zeta_{1} \frac{\partial}{\partial z}\left(\phi_{1 \alpha \alpha}+F^{-2} \phi_{1 z}\right) \\
+a_{2} k^{2} \sin k \alpha \cosh k \beta+a_{1} k^{2} \sin k \alpha \cosh k \beta \phi_{1 \alpha} \\
+2 a_{1} k \cos k \alpha \cosh k \beta \phi_{1 \alpha \alpha}-3 a_{1} k^{3} \cos k \alpha \sinh k \beta \phi_{1 \beta} \\
\left.+a_{1}^{2} k^{3} \cos k \alpha \sinh k \beta+6 a_{1} F^{-2} \cos k \alpha \cosh k \beta \phi_{1 z}\right]
\end{gathered}
$$

at $z=0$,

$$
\begin{array}{ll}
\phi_{2 \beta}=0 & \text { at } \beta= \pm 1, \\
\phi_{2 z}=0 & \text { at } z=-d .
\end{array}
$$

Similarly, the third-order differential equation and boundary conditions are brought forth by collecting all terms of order $\varepsilon^{3}$. After some simplification they are

$$
\begin{array}{r}
\phi_{3 \alpha \alpha}+\phi_{3 \beta \beta}+\phi_{3 z z}=-\left[2 k \cos k \alpha \cosh k \beta\left(a_{2} \phi_{1 z z}+a_{1} \phi_{2 z z}\right)\right. \\
\left.+a_{1}^{2} k^{2}\left(\cos ^{2} k \alpha+\sinh ^{2} k \beta\right) \phi_{1 z z}\right],
\end{array}
$$

\footnotetext{
${ }^{1}$ The expansion here has followed Ellermeier's [7] procedure for studying flows over a wavy bottom rather closely; the explicit relation between $\varepsilon$ and $a$ will be shown after the third-order differential equation is solved.
} 


$$
\begin{aligned}
\phi_{3 \alpha \alpha}+F^{-2} \phi_{3 z}=-\{ & \frac{\partial}{\partial \alpha}\left[2\left(\phi_{1 \alpha} \phi_{2 \alpha}+\phi_{1 \beta} \phi_{2 \beta}\right)+\phi_{1 z} \phi_{2 z}\right]+\zeta_{1} \frac{\partial}{\partial z}\left(\phi_{2 \alpha \alpha}+F^{-2} \phi_{2 z}\right) \\
& +\frac{\partial}{\partial \alpha}\left(\phi_{1 \alpha}^{2}+\phi_{1 \beta}^{2}+\frac{1}{2} \phi_{1 z}^{2}\right)+\zeta_{2} \frac{\partial}{\partial z}\left(\phi_{1 \alpha \alpha}+F^{-2} \phi_{1 z}\right) \\
& +\frac{1}{2}\left[\phi_{1 \alpha} \frac{\partial}{\partial \alpha}\left(\phi_{1 \beta}^{2}+\phi_{1 z}^{2}\right)+\phi_{1 \beta} \frac{\partial}{\partial \beta}\left(\phi_{1 \alpha}^{2}+\phi_{1 z}^{2}\right)\right] \\
& +\frac{1}{3}\left[\frac{\partial}{\partial \alpha}\left(\phi_{1 \alpha}^{3}\right)+\frac{\partial}{\partial \beta}\left(\phi_{1 \beta}^{3}\right)\right]+a_{3} k^{2} \sin k \alpha \cosh k \beta \\
& \left.+a_{1} f_{1}(\alpha, \beta)+a_{2} f_{2}(\alpha, \beta)+a_{1} a_{2} f_{3}(\alpha, \beta)+a_{1}^{2} f_{4}(\alpha, \beta)\right\},
\end{aligned}
$$

at $z=0$,

$$
\begin{array}{ll}
\phi_{3 \beta}=0 & \text { at } \beta= \pm 1, \\
\phi_{3 z}=0 & \text { at } z=-d,
\end{array}
$$

where $f_{1}, f_{2}, f_{3}$, and $f_{4}$ are functions of $\alpha$ and $\beta$, the explicit expressions of which are not needed since eventually $a_{1}$ and $a_{2}$ will be shown to be zero.

The first-order differential equation and boundary conditions (17)-(20) constitute an eigenvalue problem, the solution of which takes the form

$$
\phi_{1}=\sum_{n=0}^{\infty} A_{n} \sin k \alpha \cos n \pi \cosh r_{n}(z+d),
$$

where

$$
r_{n}=\sqrt{k^{2}+n^{2} \pi^{2}} \text { for } n=0,1,2,3, \ldots
$$

Physically, $r_{n}$ is the magnitude of the wave number vector in the direction normal to the wave fronts of the slanted waves with wave number $k$ in the $\alpha$-direction and $n \pi$ in the $\beta$-direction. The $A_{n}$ in (29) is determined from (18) to be

$$
A_{n}=-\frac{a_{1} k^{2}}{C_{n}^{(1)}} \int_{-1}^{1} \cos n \pi \cosh k \beta d \beta,
$$

where

$$
C_{n}^{(1)}=-k^{2} \cosh r_{n} d+F^{-2} r_{n} \sinh r_{n} d .
$$

As pointed out by Yih $[11,12]$, there are infinitely many values of the Froude number $F$ which will make $C_{n}^{(1)}$ vanish. To each of these Froude numbers, the coefficient of the amplitude, $A_{n}$, becomes infinite and resonance takes place. Accordingly, they will all be called critical Froude numbers. Let $m$ be an integer such that $C_{m}^{(1)}=0$. The critical Froude number corresponding to this mode is then given by

$$
F_{m}^{2}=\frac{r_{m}}{k^{2}} \tanh r_{m} d
$$

Equation (33) forms a set of critical Froude numbers $F_{m}$ each of which is completely determined by the parameters of the given fluid system such as the wave 
number of the side-wall corrugation $k$ and the depth of the fluid layer $d$. Equation (33) can be rewritten as

$$
\frac{k}{r_{m}} U=\sqrt{\frac{g \tanh r_{m}^{*} h}{r_{m}^{*}}},
$$

where $r_{m}^{*}$ is the dimensional total wave number in the direction normal to the slanted wave fronts. Since $k / r_{m}$ is the cosine of the angle between the $\alpha$-direction and the direction normal to the slanted wave with wave number $r_{m}^{*}$, and since the right-hand side of (34) is nothing but the wave speed of this wave, resonance takes place when the critical mean flow speed $U$ is such that its component normal to the front of the wave with wave number $r_{m}^{*}$ is equal to its speed. For a given $k, F_{m}$ increases with $m$. The smallest nontrivial critical velocity corresponds to $m=1$, which is defined as the principal critical speed, because the resonance for $m=1$ will be observed first in an experiment if the mean-flow speed, $U$, is gradually increased and because $m=1$ will also lead to the largest amplitude of the free-surface waves.

To prevent the amplitude from becoming infinite for any Froude number given by (33), one has to let $a_{1}$ be zero. Therefore, the amplitude $A_{m}$ remains undetermined. The rest of the wave components, for which $n \neq m$, need not be considered, since for them there is no resonance. This is analogous to the case where a large oscillation is excited in a water system, when the natural frequency of the system is approached by the frequency of an external oscillating force. Therefore, with the suppression of the modes other than $m$, the solution of the first-order differential system is now written as

$$
\phi_{1}=A_{m} \sin k \alpha \cos m \pi \beta \cosh r_{m}(z+d)
$$

in which $A_{m}$ is an undetermined amplitude coefficient. After obtaining $\phi_{1}$, the first-order displacement of the free surface $\zeta_{1}$ is obtained from (4) as

$$
\zeta_{1}=-A_{m} k F_{m}^{-2} \cosh r_{m} d \cos k \alpha \cos m \pi \text {. }
$$

If the above results are substituted into (21)-(24), the second-order differential system can be formed and then solved. The general solution consists of infinitely many wave components as well. However, one will find that, under resonance, the finiteness of the wave component with wave number $k$ will again force $a_{2}$ to vanish and there are at most three nonzero wave components left, which are with the transverse wave numbers $0,2 m \pi$, and $n_{0} \pi$, respectively. The $n_{0}$ is an integer such that

$$
\left(4 k^{2}+n_{0}^{2} \pi^{2}\right)^{1 / 2} \tanh \left(4 k^{2}+n_{0}^{2} \pi^{2}\right)^{1 / 2} d=4 r_{m} \tanh r_{m} d .
$$

The possible existence of such a nonzero integer for a given channel has been shown by Zhu [13]. Therefore, the solution for $\phi_{2}$ is generally

$$
\begin{aligned}
\phi_{2}=A_{m}^{2}\left[B_{0} \cosh 2 k(z+d)+\right. & B_{2 m} \cos 2 m \pi \beta \cosh r_{2 m}^{\prime}(z+d) \\
& \left.+B_{n_{0}} \cos n_{0} \pi \beta \cosh r_{n_{0}}^{\prime}(z+d)\right] \sin 2 k \alpha,
\end{aligned}
$$

in which the value of $B_{n_{0}}$ is the wave amplitude corresponding to the wave number $n_{0}$ and $r_{n}^{\prime}$ is

$$
r_{n}^{\prime}=\sqrt{4 k^{2}+n^{2} \pi^{2}}
$$


the magnitude of the wave number vector with wave number $2 k$ in the $\alpha$-direction and $n \pi$ in the $\beta$-direction. The amplitude coefficients $B_{0}, B_{2 m}$, and $B_{n_{0}}$ are completely determined ${ }^{2}$ from the boundary conditions (22). Their expressions are included in the Appendix.

The second-order displacement of the free surface can be obtained from (4) and is of the form

$$
\zeta_{2}=-A_{m}^{2} F_{m}^{2}\left[\mathbf{g}_{1}(\beta)+\mathbf{g}_{2}(\beta) \cos 2 k \alpha\right],
$$

where $\mathbf{g}_{1}(\beta)$ and $\mathbf{g}_{2}(\beta)$ are given in the Appendix.

The third-order differential system must be utilized in order to determine $A_{m}$, which remains unknown even after the second-order differential system has been solved. Upon making use of (35), (36), (38), and (40) and noting that $a_{1}$ and $a_{2}$ vanish, the third-order differential equation as well as boundary conditions $(25)-(28)$ can be simplified. The nonhomogeneous forcing term on the free surface consists of two terms now, one with wave number $k$ and another with wave number $3 k$. Correspondingly, the solution of the third-order differential system is expected to consist of two wave components. Let

$$
\begin{aligned}
\phi_{3}=\sum_{n=0}^{\infty} A_{m}^{3}[ & D_{n} \sin k \alpha \cos n \pi \beta \cosh r_{n}(z+d) \\
& \left.+E_{n} \sin 3 k \alpha \cos n \pi \beta \cosh r_{n}^{\prime \prime}(z+d)\right],
\end{aligned}
$$

where $r_{n}$ is the combined wave number for the longitudinal wave number $k$, which has been defined in (30), and $r_{n}^{\prime \prime}$ is that for the longitudinal wave number $3 k$, that is,

$$
r_{n}^{\prime \prime}=\sqrt{9 k^{2}+n^{2} \pi^{2}} \text {. }
$$

$D_{n}$ and $E_{n}$ are constants determined from the boundary conditions (26). Since the determination of $E_{n}$ does not affect the determination of the amplitude coefficient $A_{m}$, to the order of $O\left(\varepsilon^{3}\right)$, the final expression of $E_{n}$ is omitted here. For each mode $n$,

$$
D_{n}=-\frac{k^{2}}{C_{n}^{(1)}} \int_{-1}^{1}\left[A_{m}^{3} \mathbf{g}_{1}(\beta)-a_{3} k^{2} \cosh k \beta\right] \cos n \pi \beta d \beta,
$$

where $C_{n}^{(1)}$ has already been given in (32).

It is by demanding, again, the finiteness of the amplitude of the waves with the longitudinal wave number $k$ that one can determine the unknown amplitude $A_{m}$ when resonance takes place. That is, one specifies that

$$
\int_{-1}^{1}\left[A_{m}^{3} \mathbf{g}_{1}(\beta)-a_{3} k^{2} \cosh k \beta\right] \cos m \pi \beta d \beta=0,
$$

which leads to the determination of $A_{m}$ as

$$
A_{m}=k \sqrt[3]{(-1)^{m} \frac{2 a_{3} \sinh k}{r_{m}^{2} G_{8}}},
$$

\footnotetext{
${ }^{2}$ The determination of $B_{n_{0}}$ involves the usage of l'Hôspital's rule.
} 
in which $G_{8}$ is a constant defined by the integral

$$
G_{8}=\int_{-1}^{1} \mathbf{g}_{1}(\beta) \cos m \pi \beta d \beta .
$$

Since $\mathbf{g}_{1}(\beta)$ has been determined previously, the above integral can be carried out easily. The expression of $G_{8}$ is lengthy and has been included in the Appendix.

With $a_{1}$ and $a_{2}$ vanishing, it is clear now that the small number $\varepsilon$ used in the expansion (14)-(16) is nothing but $a^{1 / 3}$ if $a_{3}$ is taken to be unity and all other terms are of higher order and can be neglected. That is to say that one can take

$$
\zeta=a^{1 / 3} \zeta_{1}+a^{2 / 3} \zeta_{2}+a \zeta_{3}+\cdots,
$$

which simply shows that the amplitude of the free surface at the critical speed is of the order of the one-third power of the side-wall corrugation amplitude.

3. Discussion and summary. Upon the determination of $A_{m}$, the solution is completed as far as primary resonance is concerned. It is found that the amplitude of the free surface wave is a large but finite number whose magnitude is of the order of the one-third power of the side-wall amplitude. The successful determination of the amplitude of these stationary waves shows, through the model adopted, that the nonlinear convective acceleration terms in the Euler equations play a very important role near resonance.

To illustrate this successful determination of the amplitude of the free surface waves near resonance, a sample channel with depth of $0.4 \mathrm{~m}$, width of $1 \mathrm{~m}$, the side-wall corrugation amplitude of $0.001 \mathrm{~m}$ and side-wall corrugation wavelength of $0.4 \mathrm{~m}$ has been chosen. The critical mean flow velocities of the first four modes were determined from (33) and are listed in the first column of Table 1.

TABle 1. Prediction of the critical mean flow speed and the amplitude of the first four modes.

\begin{tabular}{ccr}
\hline Mode & $U_{\text {cr }}(\mathrm{m} / \mathrm{s})$ & \multicolumn{1}{c}{$A_{m}(\mathrm{~m})$} \\
\hline 1 & 0.820141 & -0.138460 \\
2 & 0.894305 & 0.142703 \\
3 & 0.987693 & -0.137536 \\
4 & 1.085518 & 0.131149 \\
\hline
\end{tabular}

The maximum amplitude of the free surface waves along the center line of the channel (when $\beta=0$ ) predicted by the linear solution approaches infinity, as expected and shown in Fig. 3 on next page, as the mean flow velocity $U$ approaches the critical mean flow velocity of the first mode $U_{\mathrm{cr}}$. The amplitude determined from (45) and (46) is $0.138 \mathrm{~m}$ when $U=U_{\text {cr }}$. Although the experimental data are not available at this stage to check this value, it seems to be a reasonable prediction compared to the infinitely large amplitude predicted by the linear theory. 


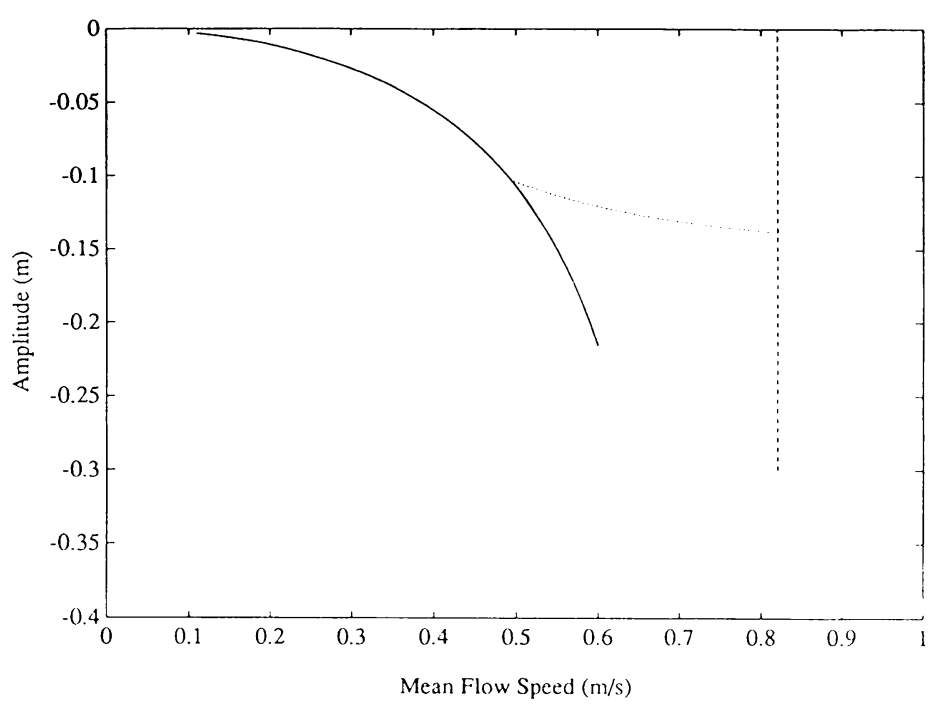

Fig. 3. The free surface amplitude predicted by the linear (solid line) and nonlinear (dotted line) theories.

Although the linear theory will not break down, theoretically speaking, until $U=$ $U_{c r}$, the amplitude predicted by the linear theory is too large to be reasonable when $U$ is close to $U_{\mathrm{cr}}$. Extra care must be taken when dealing with the upstream velocity in the intermediate range between the $U_{\mathrm{cr}}$ and a smaller $U$ at which the linear solution is valid. This can be achieved by perturbing the Froude number directly. For the convenience of making use of the nonlinear solution already presented in the above section, we expand $F^{-2}$ as a power series in $\varepsilon$, which takes the form

$$
F^{-2}=F_{m}^{-2}+\varepsilon^{2} F_{m_{2}}^{2}+O\left(\varepsilon^{4}\right) \text {. }
$$

Note that there is no first-order term in the above expansion. The expansion (48) with all odd-order terms dropped is reasonable because the physical problem is not changed when the $\varepsilon$ is changed to $-\varepsilon$. Under the expansion of the Froude number near the critical Froude number, determined from (33), only the term $F_{m_{2}}^{-2} \phi_{1 z}$ needs to be added to the third boundary condition in (26), whereas the first-order equations (17)-(20) and the second-order equations (21)-(24) remain unchanged. Following similar procedures to those demonstrated above, this additional term will eventually result in an additional term in (44). Correspondingly, the modified (45) is now of the form

$$
G_{8} A_{m}^{3}-r_{m} F_{m_{2}}^{-2} \sinh r_{m} d A_{m}=(-1)^{m} \frac{2 k^{3} \sinh k}{r_{m}^{2}} a_{3},
$$

from which the unknown amplitude coefficient $A_{m}$ is determined. The solution of the equation (49), for our test channel, is plotted out in Fig. 3 with a dotted line. As can be clearly seen, it takes care of the gap between the solution of (45) and the linear solution.

In Fig. 4, the amplitudes of the free surface under the critical Froude numbers corresponding to different modes are plotted against the variation of the wavelength of the corrugated side walls. As expected, the amplitude of the free surface waves 


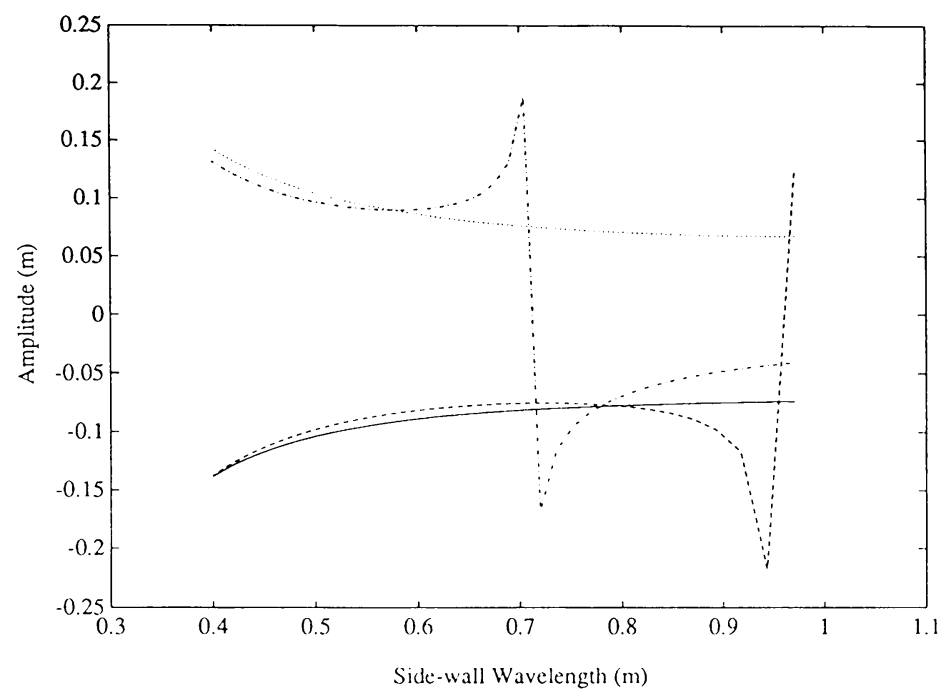

FIG. 4. The free surface amplitude predicted by the nonlinear theory vs. the variation of the wavelength of the side-wall corrugation (solid line for $m=1$, dotted line for $m=2$, dashed line for $m=3$, and dash-dotted line for $m=4$ ).

on the center cross section of the channel (i.e., the vertical plane corresponding to $\beta=0$ ) decreases as the side-wall wavelength increases. As for the limiting case, there should be no wave for a channel with two straight walls $(\lambda \rightarrow \infty)$ because the external force, which is the source of the perturbation for the waves being generated, has been removed. There is a phase shifting of the amplitude from on-phase with the side-wall corrugation $\left(A_{m}>0\right)$ to out-of-phase with the side-wall corrugation $\left(A_{m}<0\right)$ for the mode 3 (dashed line) and vice versa for the mode 4 (dash-dotted line). These phase shifts were due to the sign change of $G_{8}$ in (45), which shows the existence of higher-order resonances. The higher-order resonance takes place as both $C_{n}^{(1)}$ and $G_{8}$ vanish simultaneously for some given $k$ and $d$. However, they are not as important as the primary resonance that we are most concerned with in this paper. The determination of the amplitude coefficient, at the higher-order resonance due to the vanishing of $G_{8}$, requires that the expansion be carried to the fifth or even higher order. This expansion will involve a lot of tedious algebraic manipulation, and is left for future investigation.

Finally, the solution presented here shows that there is only one dominant wave component left. This makes sense because the neglected wave components, when resonance takes place, all have the order of magnitude $\varepsilon^{3}$ or $a$, and are thus dwarfed by the single dominant wave component emphasized in this paper.

Acknowledgment. The author wishes to thank Professor C.-S. Yih for his constant support and encouragement. Several crucial suggestions given by him made it possible to finish the work presented here. The author also wants to express his gratitude to Dr. D. A. Barry, who read the manuscript and made valuable comments. Finally, thanks are due to Miss Megan Hele, who helped draw some of the figures. 
Appendix. The solution of the second-order equation was given in (36) in terms of three amplitude coefficients, $B_{0}, B_{2 m}$, and $B_{n_{0}}$, which are determined from the given physical parameters. They are of the form

$$
B_{n}= \begin{cases}\frac{G_{1}}{C_{0}^{(2)}} & \text { if } n=0 ; \\ \frac{G_{2}}{C_{2 m}^{(2)}} & \text { if } n=2 m ; \\ \frac{(-1)^{n_{0}} 2 r_{n_{0}}^{2}\left[\left(n_{0}^{2}-4 m^{2}\right)+n_{0}^{2} G_{2}\right]}{\left.m n_{0} \pi^{2}\left(n_{0}^{2}-4 m^{2}\right)\left[\left(4 k^{2} d F_{m}^{-2}+1\right) \cosh r_{n_{0}}^{\prime} d-4 k^{2} d r_{n_{0}}^{\prime} \sinh r_{n_{0}}^{\prime} d\right)\right]} & \text { if } n=n_{0},\end{cases}
$$

in which

$$
C_{0}^{(2)}=2 k\left(F_{m}^{-2} \sinh 2 k d-2 k \cosh 2 k d\right), \quad C_{2 m}^{(2)}=-4 k^{2} \sinh ^{2} r_{m} d,
$$

and

$$
\begin{aligned}
& G_{1}=-\frac{1}{2} k\left[\frac{1}{2}\left(m^{2} \pi^{2}-3 k^{2}\right) \cosh ^{2} r_{m} d+r_{m}^{2} \sinh ^{2} r_{m} d\right], \\
& G_{2}=-\frac{1}{2} k r_{m}^{2}\left[-\frac{3}{2} \cosh ^{2} r_{m} d+\sinh ^{2} r_{m} d\right] .
\end{aligned}
$$

The second-order displacement of the free surface in (38) was given in two functions of $\beta$, which are

$$
\begin{aligned}
& \mathbf{g}_{1}(\beta)=\left(G_{3}+G_{4} \cos 2 m \pi \beta\right), \\
& \mathbf{g}_{2}(\beta)=\left(G_{5}+G_{6} \cos 2 m \pi \beta+G_{7} \cos n_{0} \pi \beta\right),
\end{aligned}
$$

where

$$
\begin{aligned}
G_{3} & =\frac{1}{8} r_{m}^{2} \\
G_{4} & =\frac{1}{8}\left(k^{2}-m^{2} \pi^{2} \cosh 2 r_{m} d\right), \\
G_{5} & =2 k B_{0} \cosh 2 k d+\frac{1}{8}\left[\left(k^{2}-m^{2} \pi^{2}\right) \cosh ^{2} r_{m} d-3 r_{m}^{2} \sinh ^{2} r_{m} d\right], \\
G_{6} & =2 k B_{2 m} \cosh r_{2 m}^{\prime} d+\frac{1}{8} r_{m}^{2}\left(\cosh ^{2} r_{m} d-3 \sinh ^{2} r_{m} d\right), \\
G_{7} & =2 k B_{n_{0}} \cosh 2 r_{n_{0}}^{\prime} d .
\end{aligned}
$$

Upon using all these predetermined constants, the $G_{8}$ in (44) and (47) can be 
zasily determined after algebraic simplification, and is

$$
\begin{aligned}
G_{8}=- & \left\{k B_{0}\left[-4 k^{2} \cosh r_{m} d \cosh 2 k d+3 k r_{m} \sinh r_{m} d \sinh 2 k d+r_{m}^{2} \frac{\cosh 2 k d}{\cosh r_{m} d}\right]\right. \\
+ & k B_{2 m}\left[-\left(r_{m}^{2}+\frac{1}{4} r_{2 m}^{\prime 2}\right) \cosh r_{m} d \cosh r_{2 m}^{\prime} d+\frac{3}{4} r_{m} r_{2 m}^{\prime} \sinh r_{m} d \sinh r_{2 m}^{\prime} d\right. \\
+ & \left.+\frac{1}{2} r_{m}^{2} \frac{\cosh r_{2 m}^{\prime} d}{\cosh r_{m} d}\right] \\
+ & {\left[\frac{1}{8} m^{2} \pi^{2} k^{2}+\frac{3}{16}\left(m^{2} \pi^{2}-k^{2}\right) r_{m}^{2}\right] \cosh ^{3} r_{m} d } \\
+ & \left.r_{m}^{2}\left(\frac{9}{16} r_{m}^{2}-m^{2} \pi^{2}\right) \cosh r_{m} d \sinh ^{2} r_{m} d-\frac{3}{32} r_{m}^{4} \frac{\cosh 2 r_{m} d}{\cosh r_{m} d}\right\} .
\end{aligned}
$$

\section{REFERENCES}

[1] J. A. Aranha, D. K. P. Yue, and C. C. Mei, Nonlinear waves near a cut-off frequency in an acoustic duct-a numerical study, J. Fluid Mech. 121, 465-485 (1982)

[2] R. Betchov, Nonlinear oscillations of a column of gas, Phys. Fluids 1, 205-212 (1958)

[3] A. M. Binnie, Self-induced waves in a conduit with corrugated walls. I. Experiments with water in an open horizontal channel with vertically corrugated sides, Proc. Roy. Soc. London Ser. A 259, $18-27(1960)$

[4] B.-T. Chu and S. J. Ying, Thermally driven nonlinear oscillations in a pipe with travelling shock waves, Phys. Fluids 6, 1625-1637 (1963)

[5] W. Chester, Resonant oscillations of water waves, Proc. Roy. Soc. London Ser. A 306, 5-22 (1968)

[6] G. Dagan and T. Miloh, Free-surface flow past oscillating singularities at resonant frequency, J. Fluid Mech. 120, 139-154 (1982)

[7] W. Ellermeier, Resonant surface waves over a wavy bottom, Personal communication, 1983

[8] A. F. Jones, The generation of cross-waves in a long deep channel by parametric resonance, J. Fluid Mech. 138, 53-74 (1984)

[9] H. Lamb, Hydrodynamics, 6th ed., Cambridge University Press, Cambridge, 1945

[10] J. R. Ockendon and H. Ockendon, Resonant surface waves, J. Fluid Mech. 59, 397-413 (1973)

[11] C.-S. Yih, Binnie waves, 14th Sympos. on Naval Hydrodynamics, Ann. Arbor, Michigan, 1982

[12] C.-S. Yih, Waves in meandering streams, J. Fluid Mech. 130, 109-121 (1983)

[13] S.-P. Zhu, Open channel flows near the resonance speed, Ph.D. thesis, Univ. Michigan, Ann. Arbor, 1987 